\title{
Experiences in obtaining prescribable and non-prescribable gluten-free products of adults with coeliac disease
}

\author{
M. Bruce ${ }^{1}$, L. White, P. Gillett ${ }^{2}$ and E. Bannerman ${ }^{1}$ \\ ${ }^{1}$ Queen Margaret University, Queen Margaret University Drive, Musselburgh, East Lothian, EH21 6UU, UK and ${ }^{2}$ Royal \\ Hospital for Sick Children, Edinburgh, UK
}

Coeliac disease (CD) is a lifelong chronic disease of the small intestine affecting approximately $1 \%$ of the population ${ }^{(1)}$. The only form of treatment is life-long adherence to a gluten-free diet (GFD), with strict adherence in adults suggested to range from 42 to $91 \%{ }^{(2)}$. It is recognised that gluten-free products (GFP) are costly for the consumer ${ }^{(3)}$ and thus within the UK individuals with CD have access to a proportion of staple GFP on prescription to assist with dietary compliance ${ }^{(4)}$. However, anecdotal evidence suggests that aspects of the current prescribing system are problematic. There is a lack of published data regarding factors that affect individuals' acquirement of GFP in both the retail market and from the UK GF prescribing system i.e. factors that potentially underpin compliance to a GFD. The aim of this study was to assess the experiences of Lothian Coeliac UK members ( $>18$ years) in obtaining both prescribable and non-prescribable GFP.

A questionnaire using both qualitative and quantitative questions was emailed in May 2010 to all Coeliac UK members ( $>18$ years, $n 863$ ), registered with a local Coeliac UK branch (Lothian, Scotland) to ascertain individuals' experiences in obtaining prescribable and non-prescribable GFP.

One hundred and thirty completed questionnaires (response rate $15 \%)$ were returned (43M: 87F, mean (SD) age 49 (14) years; median (IQR) age at diagnosis 43 (21) years; $57 \%$ did not receive regular check-ups from health-care professionals). Eighty-eight percent of participants use the current prescribing system and overall this was rated as convenient by two-thirds of the group. However, $24 \%$ found requesting the prescription a problem; $38 \%$ said that they felt the range of products available on prescription was inadequate and almost half $(49 \%)$ expressed problems when they tried to have the choice of items amended. Transporting products home was identified as a problem needing a car, or multiple trips back to the pharmacy. Once GFP were obtained, $36 \%$ had problems storing the food at home, this often resulting in products going off.

Experiences in obtaining non-prescribable GFP showed $91 \%$ of participants had changed their shopping habits since being diagnosed and $30 \%$ changed the supermarket at which they shop. Although supermarkets are the preferred location for shopping, $47 \%$ rated the supermarket as inadequate in terms of choice of GFP and $30 \%$ indicate they find food labelling difficult to understand.

This study has shown that there are inequalities in individuals' experiences of the current prescribing system of GFP. Cost, choice and availability of non-prescribable GFP are potential barriers to an 'easily-accessible' GFD.

1. Agency for Healthcare Research and Quality (2004) Evidence Report/Technology Assessment No. 104 Celiac Disease. AHRQ Publication No. 04-E029-2.

2. Hall NJ, Rubin G \& Charnock A (2009) Aliment Pharmacol Ther 30, 315-330

3. Lee AR, Ng DL, Zivin J et al. (2007) J Hum Nutr Diet 20, 423-430.

4. BDA, Coeliac UK \& PCSG (2004) Gluten-free foods: A prescribing guide. Coeliac, UK. 